\title{
Medium-range order in pyrolyzed carbon films: Structural evidence related to metal-insulator transition
}

\author{
V. Meenakshi ${ }^{\text {a) }}$ and S. V. Subramanyam ${ }^{\text {b) }}$ \\ Department of Physics, Indian Institute of Science, Bangalore 560012, India
}

\begin{abstract}
Structural properties of carbon films prepared by the pyrolysis of tetra chloro phthalic anhydride are investigated in an attempt to correlate it with the metal-insulator transition observed earlier as a function of deposition temperature, from electrical transport studies. Anomalous features are observed which evolve as the pyrolysis temperature is varied from 700 to $900{ }^{\circ} \mathrm{C}$. This includes prepeaks in the $\mathrm{x}$-ray diffraction pattern in the region less than $1 \AA^{-1}$ providing evidence for medium range order, and a broad shoulder around $1200 \mathrm{~cm}^{-1}$ in the Raman spectra, which are seen in addition to the usually observed peaks in amorphous carbon films. A simple model is proposed according to which presence of "polymeric domains" containing chains of carbon atoms with single and double bond alternation $(-\mathrm{C}=\mathrm{C}-)$, in a predominantly aromatic ring structure, could give rise to the anomalous features.
\end{abstract}

\section{INTRODUCTION}

Graphitic materials are characterized by an extended $\pi$ electron system (graphite, nanotubes, amorphous carbon, etc.). The $\pi$ electron structure allows the delocalized $\pi$ electrons to move freely throughout a molecule or crystal without distorting it, and is thought to be the origin of their novel properties (quantum effects, field emission, etc). ${ }^{1}$ In order to facilitate their use in the fabrication of devices, such as high electron mobility devices, electrodes in lithium-ion batteries, and optical or quantum devices, experiments are focused in preparing them at low temperatures $\left(<1000^{\circ} \mathrm{C}\right)$.

Pyrolysis of organic compounds under suitable conditions lead to graphite-like materials and typically their properties are strongly influenced by the starting precursor and pyrolysis temperature. Kaplan et al. ${ }^{2}$ were the first to prepare carbon films by the pyrolysis of organic anhydride precursor, specifically, pereylene tetra carboxylic di anhydride (PTCDA). These films were found to be highly conducting $(250 \mathrm{~S} / \mathrm{cm})$ in contrast with many other disordered forms of carbon. However, there was no concerted effort to study the disorder effects on the electrical transport properties of these films, although the results from conductivity measurements were indicative of a possible transition from a metallic to semiconducting behavior with pyrolysis temperature. Further investigations on several other organic anhydrides ${ }^{3}$ in which disorder is induced by ion beam bombardment, revealed large changes in conductivity. Subsequent electrical conductivity measurements undertaken on carbon films prepared from PTCDA ${ }^{4,5}$ suggested the possibility of a metalinsulator $(\mathrm{M}-\mathrm{I})$ transition as a function of pyrolysis temperature. On the whole, the interest is to explore carbon films pyrolyzed from an organic anhydride precursor, wherein the

\footnotetext{
${ }^{a)}$ Present address: Department of Physics, Texas A\&M Univeristy, College Station, Texas 77843; electronic mail: meenakshi@physics.tamu.edu

b)Author to whom correspondence should be addressed; electronic mail: svs@physics.iisc.ernet.in
}

side groups can be easily cleaved on pyrolysis to form a predominantly $s p^{2}$ bonded carbon network structure, resulting in greater conductivity. It may be noted that in the above investigations the organic anhydride precursors contain hydrogen.

Recently, we undertook similar studies on an anhydride precursor, tetra chloro phthalic anhydride, TCPA $\left(\mathrm{C}_{8} \mathrm{Cl}_{4} \mathrm{O}_{3}\right)$ that is devoid of hydrogen to ascertain the role of hydrogen. ${ }^{6}$ Remarkably, as the pyrolysis temperature is varied over a narrow range, from 700 to $900^{\circ} \mathrm{C}$, the low temperature electrical transport properties of these carbon films reveal a rich variety of phenomena largely influenced by the amount of disorder. This includes transition from an insulator to a disordered metallic state passing through a critical regime, ${ }^{6}$ and effects related to quantum transport at low temperatures such as weak-localization and electron-electron interaction that are enhanced in the disordered state: These effects were seen in the carbon films in the metallic and critical regimes. The films in the insulating regime exhibit variable range hopping conduction (VRH), with a crossover from Mott VRH to Efros-Shklovskii VRH at lower measurement temperatures. Also, for the first time, scaling in magnetoconductance ${ }^{7}$ driven by electron-electron interactions is observed. Such a variety of phenomenon is perforce a reflection of the underlying atomic structure, which is the topic of this study.

In this article we present the results of atomic structure studies on carbon films prepared from TCPA, probed using $\mathrm{x}$-ray diffraction (XRD) and Raman spectroscopy. The XRD studies reveal two additional features in the region $<1 \AA^{-1}$ that are rarely seen in amorphous forms of carbon, which provides evidence for the presence of "polymeric" domains in a predominantly aromatic ring structure. This result is further supported by Raman spectroscopic studies that reveal additional features around $1200 \mathrm{~cm}^{-1}$ and some previous neutron diffraction studies. ${ }^{8}$ The XRD pattern in the present study is also strikingly different from the carbon films that are prepared by pyrolysis (under similar conditions) of anhydride precursors such as PTCDA and maleic anhydride con- 
taining hydrogen, in which only a broad amorphous hump around $22^{\circ}-23^{\circ}$ is seen. ${ }^{5}$

\section{EXPERIMENTAL DETAILS}

\section{A. Carbon film deposition}

The carbon films have been prepared by vapor phase pyrolysis and the experimental setup used is similar to that of Kaplan and coworkers. ${ }^{2}$ Basically, pyrolysis is carried out in a quartz tube passing through two furnaces, as shown in Fig. 1 of Ref. 9, except that the outlet of the quartz tube is connected to a bladder. One of the furnaces (A) is used for heating the precursor material and the other (B) is used for controlling the substrate or deposition temperature. Onehundred milligrams of the precursor, TCPA (Fluka chemicals), is taken in a clean fused quartz tube, of $10 \mathrm{~mm}$ inner diameter, which is closed at one end; the open end is connected to a gas-collecting bladder. The precursor is held at the closed end by high temperature glass wool and the quartz tube is evacuated ( $10^{-4}$ Torr) to avoid any possible contamination by air. When the temperature in furnace $\mathrm{B}$ is stabilized at the desired preparation temperature $\left( \pm 3{ }^{\circ} \mathrm{C}\right)$ using a proportional-integral-derivative (PID) controller, furnace $\mathrm{A}$ is heated up gradually at $2{ }^{\circ} \mathrm{C} / \mathrm{min}$ to a temperature above the boiling point of the precursor $\left(373^{\circ} \mathrm{C}\right)$. The precursor evaporates and passes through the hot zone in furnace $\mathrm{B}$. Here it undergoes pyrolysis, depositing carbon on the substrates as well as on the inside wall of the quartz tube. The gases that are liberated collect outside the hot zone in the bladder. The amount of gas evolved is less and can be accommodated easily in the bladder without any significant increase in pressure inside the tube or bladder. Therefore pyrolysis and later annealing occur at ambient pressure except in the beginning when there is a vacuum inside. The evaporation of the precursor is completed in about $2 \mathrm{~h}$. The high temperature in furnace B is maintained for $30 \mathrm{~min}$, so that the deposited films are annealed as well. However, if the annealing time is increased further, the films peel off from the substrate easily, precluding electrical measurements. The above conditions for film deposition were maintained throughout the present study. After the annealing period, the tube is cooled to room temperature at a rate of $10^{\circ} \mathrm{C} / \mathrm{min}$. Free-standing films are also formed inside the tube, which are collected by breaking the tube and are in the form of curved fragile shiny flakes. In the present investigation, the precursor is pyrolyzed at three different temperatures, 700,800 , and $900{ }^{\circ} \mathrm{C}$ and the resultant carbon films are labeled T700, T800, and T900, respectively.

\section{B. X-ray measurements}

$\mathrm{X}$-ray diffraction patterns are recorded using a powder diffractometer (Siemens D5005) using Ni filtered $\mathrm{Cu} K \alpha$ radiation $(\lambda=1.5418 \AA)$ with $I=25 \mathrm{~mA}$ and $V=30 \mathrm{kV}$. Intensity is recorded from $2 \theta=2^{\circ}$ to $90^{\circ}$, in steps of $0.1^{\circ}$ allowing a step time of $15 \mathrm{~s}$, in the reflection mode, on powdered samples. It was observed that the x-ray diffracted intensity from the carbon films deposited on the quartz substrate had contribution from the substrate, as the thickness of the films are only about 1 to $2 \mu \mathrm{m}$ and the sample density is only about $1.6-1.8 \mathrm{~g} / \mathrm{cm}^{3}$. Also, it was found that it is not possible to deduct the contribution of the substrate to the scattered intensity, as the scattering by the substrate with and without the film on it is different. This problem is overcome by taking a larger amount of powdered carbon film $(\sim 300 \mathrm{mg})$ in a sample holder, ensuring negligible contribution from the holder. These samples were prepared under identical conditions as those for electrical measurements, except that no substrates were used. The quantity of film obtained from each preparatory run is small $(\sim 10 \mathrm{mg})$ and hence the runs had to be repeated many times to prepare the required amount for each of the three samples. The free films formed in the quartz tube are powdered and used for the measurements and the data are collected for T700, T800, and T900.

\section{Raman spectroscopic measurements}

The Raman spectra were obtained using a Renishaw Raman-scope spectrometer, which has a helium-neon (632.8 $\mathrm{nm})$ laser as the source. ${ }^{10}$ The microscopic attachment is an Olympus BH2 system. The spectra are recorded over the range $500-2500 \mathrm{~cm}^{-1}$ with a spectral resolution better than 2 $\mathrm{cm}^{-1}$. The samples used for this study are carbon films deposited on quartz substrates (T700, T800, and T900).

\section{RESULTS}

Rutherford backscattering (RBS) experiments ${ }^{6}$ performed to estimate the chlorine concentration in the carbon films revealed no $\mathrm{Cl}$ (within the detection limits of the RBS system) in T900. T700 and T800 were found to have 4600 and $6600 \mathrm{ppm}$ (error $\pm 10 \%) \mathrm{Cl}$, respectively.

The powder XRD pattern for the carbon samples (T700, T800, and T900) are shown in Fig. 1. The data exhibits more features than what is found usually in highly disordered forms of carbon, where only a broad hump is seen around $20^{\circ}$. The structure factor, $S(Q)$ (Fig. 2) was extracted from the raw data by correcting for Compton scattering, absorption, and polarization following the procedure outlined by Warren. ${ }^{11}$ The peak positions in the experimental curve (Fig. 1) and the corresponding particle sizes were determined by fitting to a Gaussian distribution. Having known the peak position, the interlayer spacing " $d$ " is then calculated using Bragg's formula, $d=\lambda / 2 \sin \theta$. The interlayer spacing along with the full width measured at half maximum (FWHM) of the peak intensities enable us to determine the correlation length $L$, which in other words is the number of stacked layers along the $c$ direction and the layer diameter along the $a$ direction (lateral extent of graphene layers), based on the Scherrer formula,

$$
L=\frac{K \lambda}{B_{2 \theta} \cos \theta},
$$

where $\lambda$ is the wavelength of the $\mathrm{x}$ rays used (1.5418 $\AA$ ), $B_{2 \theta}$ is the FWHM, and $K$ is a constant ${ }^{11}$ which is equal to 0.94 and 1.84 for the calculation of correlation length along the $c$ and $a$ directions, respectively. Preliminary neutron diffraction experiments ${ }^{8}$ indicated that these carbon films are predominantly $s p^{2}$ bonded and hence here an attempt is made to compare the XRD pattern with that of graphite. It can be 


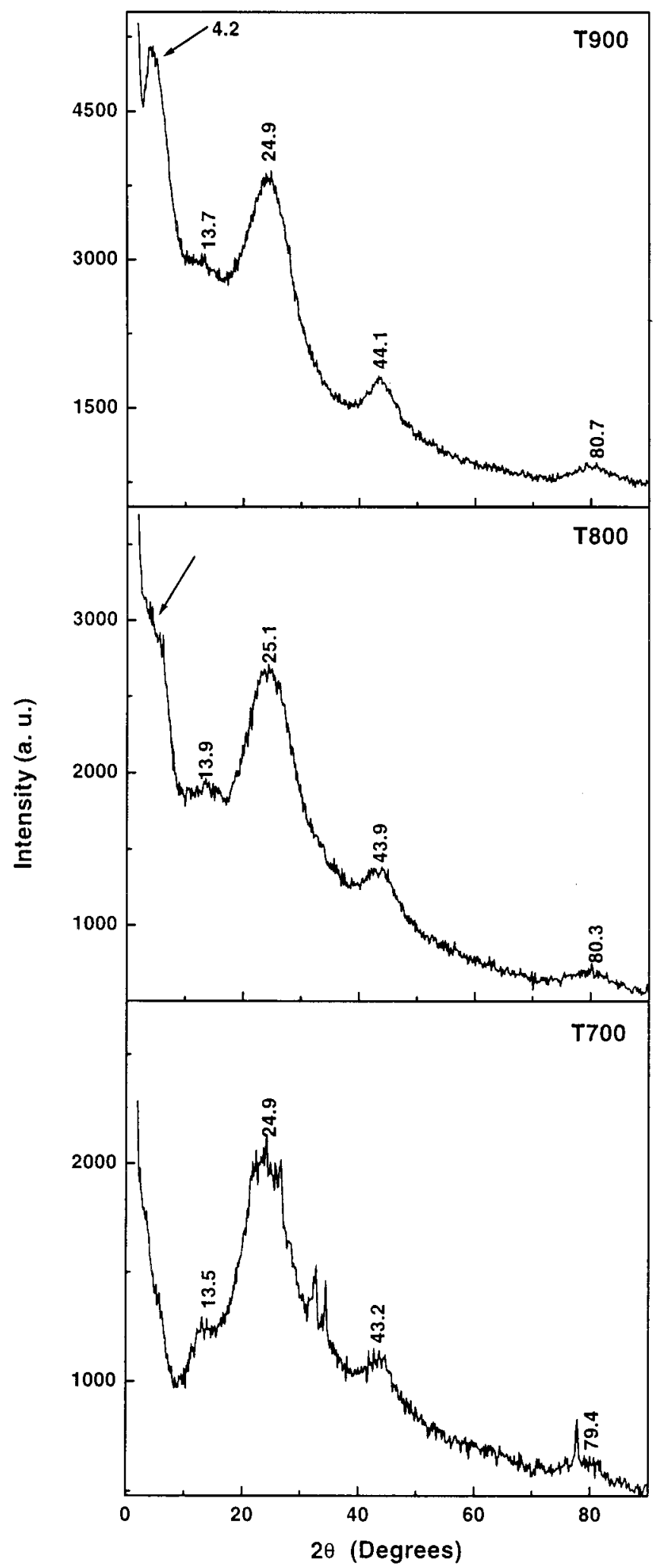

FIG. 1. Raw XRD data of T700, T800, and T900 with the peak positions (after suitable corrections) indicated. The arrows in T800 and T900 point to the development of prepeak.

seen from Fig. 1 that the broad peaks seen around $25^{\circ}, 44^{\circ}$, and $80^{\circ}$ can be identified with (002), (100)/(101), and (110) reflections in graphite, respectively.

Table I lists the position of the peaks along with the correlation lengths. The position of the peak corresponding

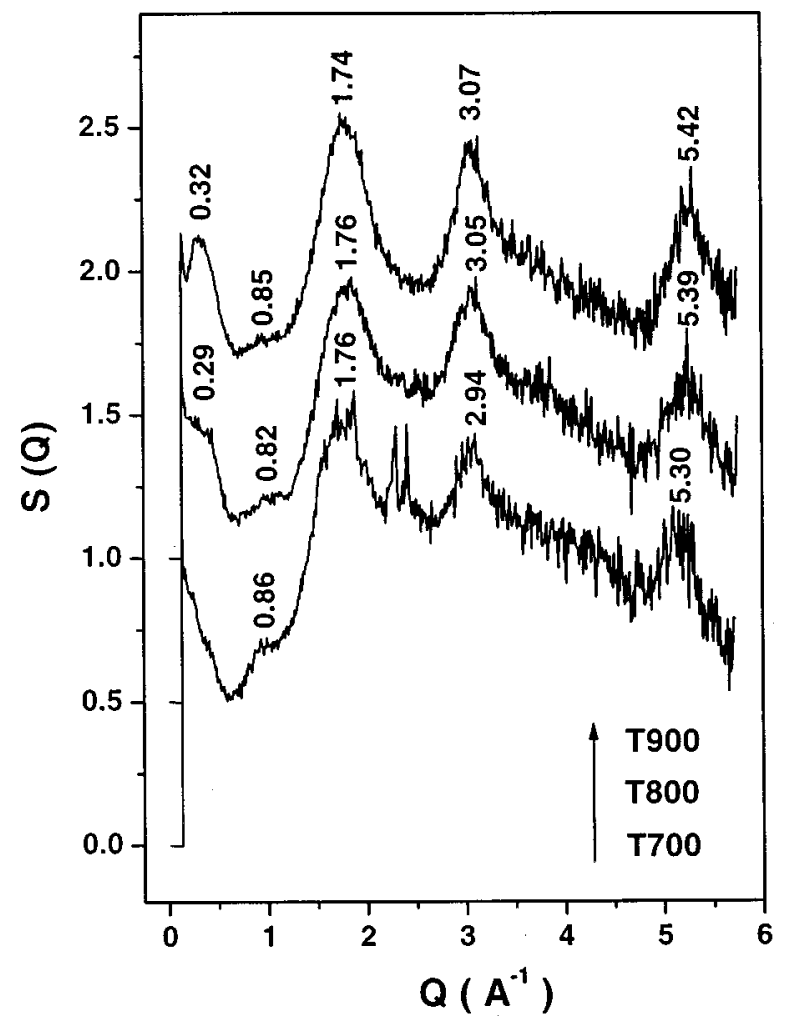

FIG. 2. Structure factor of T700, T800, and T900. T800 and T900 are shifted by 0.5 and 1.0 units, respectively, for clarity.

to the (002) plane in graphite does not vary much with increasing pyrolysis temperature. The second feature (Fig. 1) related to the $(100)$ or (101) peak of graphite shifts with increase in pyrolysis temperature from $43.2^{\circ}$ (T700) to $44.1^{\circ}$ (T900). In T800 and T900, it seems that this peak has contributions from both (100) and (101) planes. It is possible to deconvolute this broad feature, as has been done, for example, in the case of graphite nanoparticles. ${ }^{12}$ However, even after this procedure the situation remains ambiguous. Assuming that this peak arises solely due to (100) planes, the peak width and correlation length have been estimated and are shown in Table I. This is justified since contributions from (101) usually become undetectable even in the presence of a small misalignment between the graphite planes. The third feature pertaining to the (110) plane in graphite evidently

TABLE I. Parameters evaluated from the XRD data.

\begin{tabular}{lccc}
\hline \hline Sample & Peak center $(\mathrm{deg})$ & Peak width $(\mathrm{deg})$ & Correlation length $(\AA)$ \\
\hline$(002)$ & & & \\
T700 & 24.9 & 8.9 & 9.4 \\
T800 & 25.1 & 7.4 & 10.9 \\
T900 & 24.9 & 7.3 & 11.5 \\
$(100) /(101)$ & & & \\
T700 & 43.2 & 11.4 & 7.8 \\
T800 & 43.9 & 5.1 & 17.4 \\
T900 & 44.1 & 4.4 & 20.2 \\
$(110)$ & & & \\
T700 & 79.4 & 13.1 & 16.1 \\
T800 & 80.3 & 9.0 & 23.6 \\
T900 & 80.7 & 8.1 & 26.3 \\
\hline \hline
\end{tabular}


shifts, with increase in pyrolysis temperature, from $79.4^{\circ}$ (T700) to $80.7^{\circ}$ (T900). The general trend in all the samples is that with increase in pyrolysis temperature (Table I), the peak width decreases and the correlation length increases.

Apart from the usual graphitic peaks, significantly, the following two additional features are observed: (1) A broad weak shoulder is seen around $13.5^{\circ}-14^{\circ}$ (corresponding to $d$ of $\sim 6.5 \AA$ ). This feature which is prominent in T700, progressively weakens in T800 and T900, (2) Also, a weak feature seen at a low angle of about $4.2^{\circ}$ (corresponding to $d$ $=21 \AA$ ), gains strength with increasing pyrolysis temperature and becomes clearly visible in T900 (as shown by an arrow in Fig. 1). As the peak at $4.2^{\circ}$ gains prominence with increasing pyrolysis temperature, the shoulder centered around $13.5^{\circ}-14^{\circ}$ becomes weak and this reversal of the dominance in peak height is seen to occur at T800. It must be noted here that the two sharp features seen in T700 between $31.5^{\circ}$ and $32.5^{\circ}$ pertain to the precursor, TCPA, and are absent in samples prepared at higher pyrolysis temperatures.

Figure 2 shows the $S(Q)$ for the samples. In this figure, the weak features that are seen at higher angles in Fig. 1 are magnified because of the normalization of the intensity by the square of the scattering amplitude. The peaks positioned at $1.74-1.76,2.94-3.07$, and $5.30-5.42 \AA^{-1}$ correspond to those of $(002),(100) /(101)$, and (110) in graphite, respectively, which are slightly shifted in position from that of graphite. No attempt is made to Fourier transform the $S(Q)$ to obtain the radial distribution function because of the limited $Q$ space data available.

Figure 3 shows the raw data (open circles) for the Raman spectra of the carbon films deposited on quartz substrate. In each of the spectrum, the background intensity decreases almost linearly and it is deducted after fitting it to a third order polynomial. The observed line shapes are symmetric and are deconvoluted into four Gaussians for T700. For T800 and T900, in order to obtain the best fit, a fifth Gaussian peak is introduced. In the case of T900, a broad shoulder is visible around $1200 \mathrm{~cm}^{-1}$. The deconvoluted peaks are shown below each of the spectrum. While fitting, all three parameters, namely the peak position, the height, and width were allowed to vary. Also, the analysis was repeated by fixing the peak positions and allowing the other parameters to vary and the results in the two cases were almost the same. We report in Table II the quantities obtained by varying all three parameters. The best fits obtained for the data are shown as continuous lines in Fig. 3. Although only two peaks, corresponding to the disordered $(D)$ peak (1350 $\left.\mathrm{cm}^{-1}\right)$ and graphitic $(G)$ peak $\left(1580 \mathrm{~cm}^{-1}\right)$, are evident in the raw data, we would like to emphasize that a two-symmetricline fit of Gaussians is not found to be suitable, which has also been eloquently discussed in Ref. 13. After the overall fit, two/three more peaks appear as weak features.

Table II lists the relevant quantities derived from the fits for T700, T800, and T900: The position of the $D\left(P_{D}\right)$ and $G\left(P_{G}\right)$ peaks, width of the $D\left(\omega_{D}\right)$ and $G\left(\omega_{G}\right)$ peaks, and the ratio of intensities of the $D\left(I_{D}\right)$ peak to $G\left(I_{G}\right)$ peak. The effects of the increase in pyrolysis temperature are: (i) The position as well as the width of the $G$ peak does not change much; (ii) The width of the $D$ peak decreases-the change is

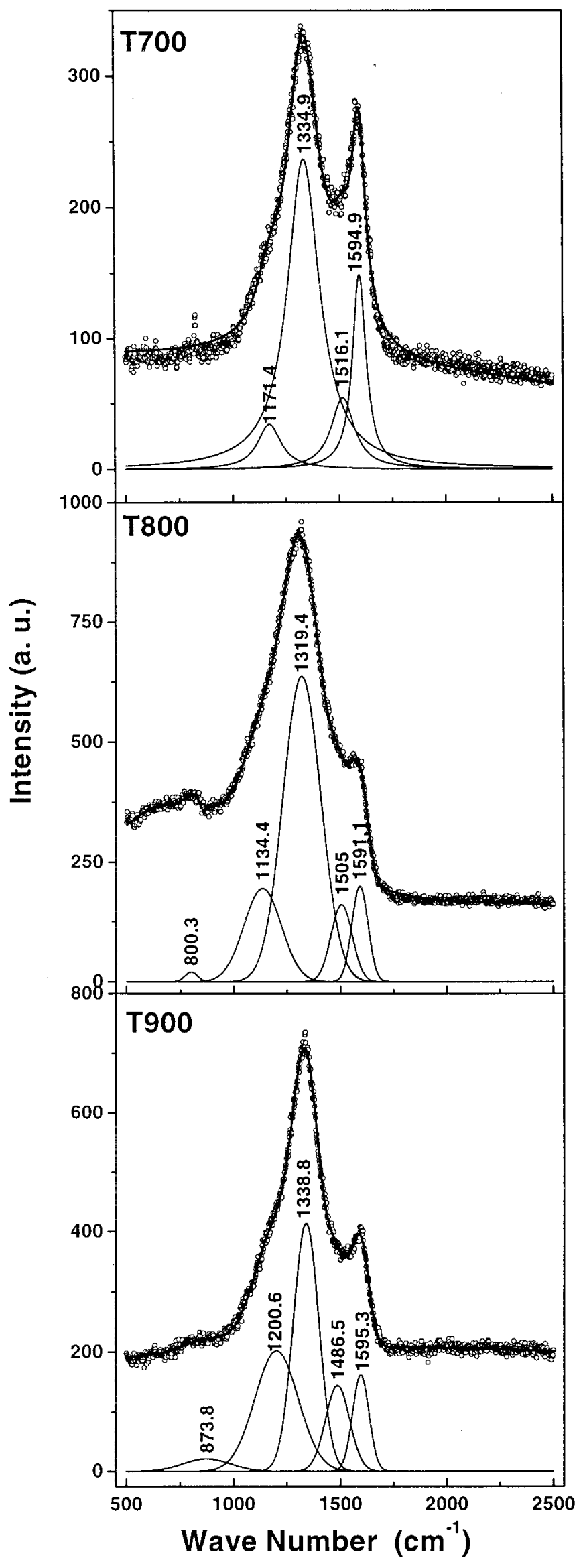

FIG. 3. Raman spectroscopic data of T700, T800, and T900. The open circles indicate the data point and the continuous line is the fit. The deconvoluted peaks along with their positions are shown below each of the spectra. 
TABLE II. Parameters evaluated from Raman spectra.

\begin{tabular}{lccccc}
\hline \hline Sample & $\begin{array}{c}P_{D} \\
\left(\mathrm{~cm}^{-1}\right)\end{array}$ & $\begin{array}{c}P_{G} \\
\left(\mathrm{~cm}^{-1}\right)\end{array}$ & $\begin{array}{c}\omega_{D} \\
\left(\mathrm{~cm}^{-1}\right)\end{array}$ & $\begin{array}{c}\omega_{G} \\
\left(\mathrm{~cm}^{-1}\right)\end{array}$ & $\begin{array}{c}\left(I_{D} / I_{G}\right) \\
(\text { a.u. })\end{array}$ \\
\hline T700 & 1334 & 1594 & 182 & 76 & 1.59 \\
T800 & 1319 & 1591 & 178 & 75 & 3.17 \\
T900 & 1338 & 1595 & 120 & 77 & 2.55 \\
\hline \hline
\end{tabular}

small while going from T700 to T800 but the decrease is larger between T800 and T900; (iii) $D$ peak position is shifted to a lower frequency in T800; and (iv) The $I_{D} / I_{G}$ ratio is maximum for $\mathrm{T} 800$.

\section{DISCUSSION}

\section{A. X-Ray diffraction}

First, let us see how atomic disorder and pyrolysis temperature affects the peak positions in the XRD pattern in comparison with that found in graphite. The peak around $25^{\circ}$, observed in all the samples, points to a layered structure in these films, though it occurs at a lower angle compared to that in graphite (Fig. 1). The interlayer distance between graphene sheets are estimated as $\sim 3.56 \AA$, which is considerably higher than that observed in graphite $(3.355 \AA)$ : We understand this as due to the reduction in packing efficiency because of disorder. The misalignment of graphite-like planes increases the separation between them, causing the peak to shift to lower angles. However, the position of this peak does not vary much with pyrolysis temperature indicating that the alignment of the planes is not significantly affected by pyrolysis temperature. The presence of this broadened peak points to the possible existence of small graphitic clusters. This is in conformity with the conducting nature ${ }^{6}$ of the films and is also supported by Raman measurements on these films. ${ }^{10}$ The present results also indicate an increase in size of these clusters, since the peak width decreases as the pyrolysis temperature increases (Table I).

Now, attention is focused on the two features observed at low $Q$ values. We discuss it from the viewpoint of $S(Q)$ of other forms of amorphous carbon, particularly in the domain $<1 \AA^{-1}$ (Table III). In the present study, as the pyrolysis temperature increases, the feature at $0.32 \AA^{-1}$ in the $S(Q)$ becomes prominent as seen from Fig. 2. Also, all the samples show a weak broad feature centered around $0.82-0.86 \AA^{-1}$.
In general, the origin of peaks occurring at $S(Q)<1 \AA^{-1}$, in the carbon system (Table III), have been traced to the presence of voids or medium range order (MRO) of atoms. ${ }^{18,19}$ Another inference that can be drawn from Table III is that low-angle features are commonly observed in polymer-like amorphous carbon films. Taking a cue from these studies, we associate such features seen in the present study with medium-range ordering of atoms. Further we attribute this order to the presence of polymer-like domains, for which further evidence is provided by the Raman spectroscopic measurements to be discussed next and also from neutron diffraction experiments. ${ }^{8}$

The presence of these polymeric domains can obstruct the tendency towards graphitization (the process where randomly stacked defective graphite sheets are converted to properly stacked crystalline graphite when heat treated to $\sim 2500{ }^{\circ} \mathrm{C}$ ); this hypothesis is supported by the position of the peak around $\sim 24.9^{\circ}$ (Fig. 1), which does not move towards higher angles (better alignment of graphite planes) with increasing pyrolysis temperature. However, the peak width decreases with increasing pyrolysis temperature signifying an increase in correlation length (size of graphitic clusters).

\section{B. Raman spectroscopy}

As already mentioned, major features in the Raman spectra of amorphous carbon films are the " $G$ " mode (characteristic of intralayer vibration), and in microcrystalline and disordered graphite, a " $D$ " mode which is inactive for an infinite layer and is activated by the absence of phonon wave-vector (k) conservation, due to the presence of disorder. In general, the positions, widths, and relative intensities of these two features are found to vary with deposition conditions and film properties. ${ }^{20-22}$

The peak position in the Raman spectra yields information concerning the nature of bonding and bond-angle disorder. $^{20}$ It is evident from the results of the carbon films under study that graphitic order ( $G$-peak width and position) is not affected by variation in pyrolysis temperature, corroborating the results from XRD. The significant reduction in the width (Table II) of the $D$ peak (characterizes disorder) in T900 indicates the reduction of bond-angle disorder, which can be associated with the transition to metallic state.

TABLE III. Peaks in $S(Q)$ below $1 \AA^{-1}$ found in a few carbon forms.

\begin{tabular}{|c|c|c|c|}
\hline $\begin{array}{l}\text { Peak position } \\
\qquad\left(\AA^{-1}\right)\end{array}$ & Carbon form & Origin & Reference \\
\hline $0.2-0.5,0.8$ & $\begin{array}{l}\text { Polymer-like amorphous } \\
\text { carbon films }\end{array}$ & $\begin{array}{c}\text { Medium range order generated } \\
\text { in } S(Q) \text { of nano-crystalline } \\
\text { polypropylene }\end{array}$ & 14 \\
\hline 0.4 & Carbon nanotubes & Corresponds to (10) reflection & 15 \\
\hline 0.9 & $\begin{array}{c}\text { Doped conducting } \\
\text { polymers (doped polypyrrole) }\end{array}$ & $\begin{array}{l}\text { Corresponds to }(010) \text { reflection and } \\
\text { the evolution indicative of decrease } \\
\text { in disorder in the direction of polymer } \\
\text { chain, as samples become metallic }\end{array}$ & 16 \\
\hline$<1$ & $\begin{array}{l}\text { Amorphous hydrogenated } \\
\text { carbon films }\end{array}$ & Voids/medium range order & 17,18 \\
\hline
\end{tabular}




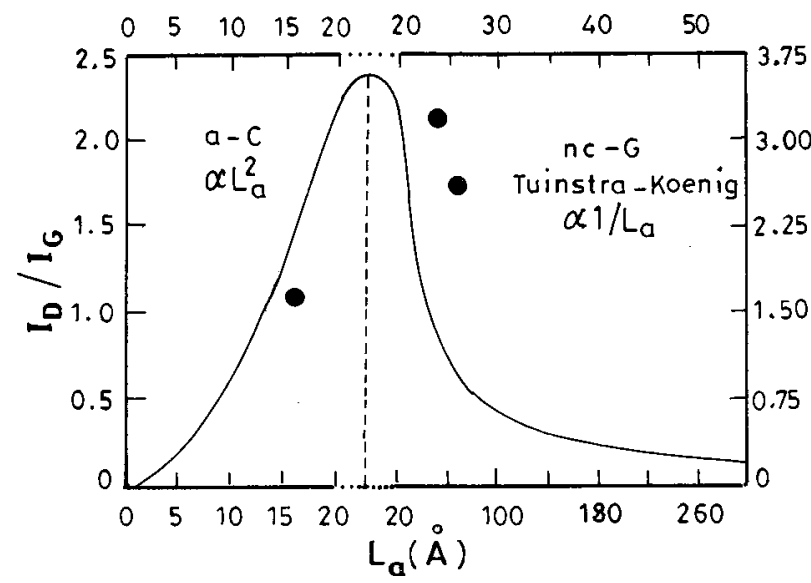

FIG. 4. The continuous line indicates the variation in the $I_{D} / I_{G}$ ratio with crystallite size $\left(L_{a}\right)$ for micro/nanocrystalline graphite and amorphous carbon (taken from Ref. 13). The closed circles correspond to data for T700, T800, and T900, which are with respect to the top $(x)$ and right $(y)$ axes.

\section{Variation in $I_{D} / I_{G}$ with pyrolysis temperature}

We now explain the dependence of the $I_{D} / I_{G}$ ratio (Table II) on pyrolysis temperature. Figure 4 taken from the article by Ferrari and Robertson ${ }^{13}$ depicts the general variation (shown by a continuous line) of $I_{D} / I_{G}$ as a function of crystallite size, in micro/nanocrystalline graphite and amorphous carbon; the closed circles marked in this graph correspond to T700, T800, and T900 (taken from Tables I and II). In amorphous carbon, such as the samples in the present study, the $I_{D} / I_{G}$ ratio increases with crystallite size. Interestingly, this implies that the $I_{D} / I_{G}$ ratio increases with order. At the outset, this sounds odd, but is understood in the following way: Structurally, $I_{D}$ ( $D$ peak) is related to the presence of sixfold aromatic rings, while the $G$ peak occurs at all $s p^{2}$ sites and does not require the presence of sixfold rings. In the presence of disorder that reduces the crystallite sizes to values lower than $20 \AA$, aromatic clusters become smaller and distorted; and consequently they open up. This reduction in the number of aromatic rings decreases $I_{D}$ with respect to $I_{G}$. Coming to our present study, in $\mathrm{T} 700$, the crystallites are smaller and fewer and therefore the $I_{D} / I_{G}$ ratio is smaller. Now, as the pyrolysis temperature is increased from 700 to $800^{\circ} \mathrm{C}$, there is an increase in crystallite size/number ${ }^{20}$ and hence the contribution to the $D$ band increases which leads to an increase in the $I_{D} / I_{G}$ ratio. In the $k$-space language, the $I_{D} / I_{G}$ ratio is proportional to the ratio of momentum nonconserving to momentum conserving phonons contributing to the Raman spectrum. In Fig. 4, it can be seen that roughly beyond a crystallite size of $20 \AA$, the $I_{D} / I_{G}$ begins to fall. This is because, as crystallite size grows, the number of momentum conserving phonons increases as compared to momentum nonconserving phonons. This explains the decrease in $I_{D} / I_{G}$ in T900. Moreover, we estimated the crystallite size from XRD for T800 to be $\sim 23.6 \AA$, which is close to the value in the above graph, where the $I_{D} / I_{G}$ ratio is predicted to decrease. This result is also in conformity with the model of Wada et al. ${ }^{23}$ We emphasize that this behavior of $I_{D} / I_{G}$ is in stark constrast to what is observed in graphite, where the $D$ peak indicates disorder. ${ }^{13}$ This trend has also been noted in ion-beam irradiated glassy carbon ${ }^{24}$ and in sputtered carbon films from camphoric carbon. ${ }^{25}$ Overall, in the present carbon films, an increase in pyrolysis temperature leads to increased order (growth of crystallites and/or removal of bond angle disorder), and is in harmony with the results obtained from XRD.

\section{A plausible model}

From the results and fits presented in Figs. 2 and 3, it is clear that the carbon films in the present study have structural features slightly different from the others. X-ray diffraction shows unusual low $Q$ features $\left(<1 \AA^{-1}\right)$, signifying MRO engendered by polymeric domains. Though the characteristic $D$ and $G$ peaks are clearly present in the Raman spectra, additional features around $800 \mathrm{~cm}^{-1}$ and $1135-1200$ $\mathrm{cm}^{-1}$ are seen. Table IV furnishes the position and origin of a few Raman peaks other than the usually observed $D$ and $G$ peaks in some carbon forms. Interestingly, a predominant peak has been reported ${ }^{10}$ around $1175 \mathrm{~cm}^{-1}$ in carbon films prepared by pyrolysis of maleic anhydride, and the 1150 $\mathrm{cm}^{-1}$ Raman mode in nanocrystalline diamond ${ }^{29}$ is assigned to trans-polyacetylene (trans-PA), the origin of which is related to the chemical vapor deposition mechanism. This suggests that the carbon films (T700, T800, and T900) may contain polymeric chains of carbon atoms with single and double bond alternation $(-\mathrm{C}=\mathrm{C}-$ ), in addition to the aromatic ring network. ${ }^{31}$ Whereas in conjugated polymers, this kind of alternating chain structure is stabilized by the presence of hydrogen, the samples in the present study are devoid of hydrogen and hence the presence of $-\mathrm{C}=\mathrm{C}$ - chains has to be stabilized with some sort of networking with aromatic ring domains. The peak position recognized to correspond to the polymeric chains varies between 1135 and 1200 $\mathrm{cm}^{-1}$ with pyrolysis temperature and a plausible reason for this could be the variation of the chain length. The companion mode ${ }^{19,29}$ found around $1500 \mathrm{~cm}^{-1}$ in the Raman spectra, though weak, is again indicative of MRO.

The presence of small polymeric domains in the sample is also apparent from our preliminary neutron diffraction measurements, ${ }^{8}$ wherein a striking similarity is obvious between the structure factor of the carbon film prepared at $900^{\circ} \mathrm{C}$ and that of a theoretical calculation for a carbon phase containing very small polymeric domains as in an anthracene mesophase heat treated up to $600{ }^{\circ} \mathrm{C}^{32}$

The evolution of a peak at $0.32 \AA^{-1}$ in the XRD and the observation of a shoulder at $1200 \mathrm{~cm}^{-1}$ in the Raman spectra are features that become prominent only when the sample is prepared at a pyrolysis temperature of $900^{\circ} \mathrm{C}$. Probably, this is the temperature that favors to a certain extent the growth of polymeric domains (like conjugated carbon chains) from the nonpolymeric precursor, TCPA. It is interesting to note here that in the presence of such polymeric domains a metallic conductivity has been suggested, ${ }^{33}$ which we do observe in T900. However, these polymeric domains can only be present to a small extent, as the aromatic ring structure has higher thermodynamic stability. 
TABLE IV. Relavant peaks (non- $D$ and $G$ peaks) in Raman spectra of some carbons.

\begin{tabular}{|c|c|c|c|}
\hline $\begin{array}{l}\text { Peak position } \\
\quad\left(\mathrm{cm}^{-1}\right)\end{array}$ & Carbon form & Origin & Reference \\
\hline 600,1140 & Hydrogen-free carbon films & Mixed $s p^{2}-s p^{3}$ hybridization & 23 \\
\hline 700 & Sputter grown $a-\mathrm{C}: \mathrm{H}(\mathrm{N})$ & $\begin{array}{l}\text { Structural disorder arising due } \\
\text { to graphite microcrystallites }\end{array}$ & 26 \\
\hline 800 & $\begin{array}{l}\text { Conjugated polymers like } \\
\text { polyacetylene (PA) }\end{array}$ & $\begin{array}{l}\text { Not identified but attributed } \\
\text { to disorder }\end{array}$ & 27 \\
\hline $1130-1200$ & Conjugated polymers like PA & $\begin{array}{l}\mathrm{C}-\mathrm{C} \text { bond stretching and } \mathrm{CH} \\
\text { in-plane bending }\end{array}$ & 27 \\
\hline 1150 & $\begin{array}{l}\text { Ion implanted highly oriented } \\
\text { pyrolytic graphite (HOPG) }\end{array}$ & $\begin{array}{l}\text { A kind of hydrogenated-like } \\
\text { carbon with alternate } \mathrm{C}=\mathrm{C} \\
\text { and } \mathrm{C}-\mathrm{C} \text { bonding }\end{array}$ & 28 \\
\hline $\begin{array}{l}1150 \\
\text { (dispersion of } 50-100)\end{array}$ & Nanocrystalline diamond & $\begin{array}{l}\text { Trans PA, corresponds to the } \\
\text { sum and difference combinations } \\
\text { of } \mathrm{C}=\mathrm{C} \text { chain stretching and } \\
\mathrm{CH} \text { wagging modes }\end{array}$ & 29 \\
\hline 1170 & Glassy carbon & High phonon density of states & 30 \\
\hline 1175 & $\begin{array}{l}\text { Pyrolysis of maleic anhydride } \\
\text { at } 800^{\circ} \mathrm{C}\end{array}$ & $\begin{array}{l}\text { Presence of polymeric chains } \\
\text { of carbon atoms, }-\mathrm{C}=\mathrm{C}-\text {, in } \\
\text { addition to aromatic rings }\end{array}$ & 10 \\
\hline $\begin{array}{l}1450 \\
\text { (dispersion of } 50-100)\end{array}$ & Nanocrystalline diamond & $\begin{array}{c}\text { Companion mode of } 1150 \mathrm{~cm}^{-1} \text {, } \\
\text { usually obscured by the tail of } \\
G \text { peak }\end{array}$ & 29 \\
\hline 1500 & Ion implanted HOPG & $\begin{array}{l}\text { Amorphous-like structure } \\
\text { with } \mathrm{C}=\mathrm{C} \text { bonding }\end{array}$ & 28 \\
\hline
\end{tabular}

\section{Some remarks}

The above picture of polymeric domains embedded in an aromatic network is also consistent with a view ${ }^{34}$ prevalent in covalent glass network, that soft domains in a rigid network lead to the formation of a strain-free structure that is thermodynamically metastable. In this context, studying the evolution of nano-indentation hardness with pyrolysis temperature and understanding the results on the basis of constraint theory for mechanical networks ${ }^{35}$ might prove insightful. Also, it is well-known that mechanical properties of covalent networks are affected by one-fold coordinated halogen atoms: Such atoms interrupt network connectivity and modify the microstructure. ${ }^{36}$ In this context, although the percentage of chlorine atoms present in the carbon films under study appears small $(<1 \%)$, a comprehensive investigation along these lines will be fruitful.

The signature of the M-I transition in pyrolyzed amorphous carbon films prepared at temperatures less than $1000{ }^{\circ} \mathrm{C}$ is in itself a rare and a significant result. The transition is subtle (similar to continuous transitions in randomly doped impurity band semiconductors ${ }^{37}$ such as $\mathrm{Si}: \mathrm{P}$ ) and the difference between samples prepared at 750 and $700{ }^{\circ} \mathrm{C}$ is not as apparent as in samples prepared at 700 and $800^{\circ} \mathrm{C}$. Our overall goal is to understand the structure across the M-I transition observed by us through resistivity studies in these samples ${ }^{6}$ and not so much on the critical region centered around $800^{\circ} \mathrm{C}$.

\section{SUMMARY AND CONCLUSIONS}

$\mathrm{X}$-ray diffraction measurements performed to discern the difference in the structure of amorphous carbon films with pyrolysis temperature of the organic anhydride precursor, TCPA, show three broad peaks which correspond to those found in graphite. In these measurements, two additional peaks are found in the region $<1 \AA^{-1}$, indicating mediumrange ordering of atoms, whose origin is traced to the presence of polymeric domains in a predominantly aromatic ring structure. Especially, the evolution of the prepeak at 0.32 $\AA^{-1}$ with increasing pyrolysis temperature, that becomes prominent in T900, is correlated with the previously observed metallic nature of the sample. This conclusion finds support from the structure studies on doped conducting polymers where a M-I transition is seen. ${ }^{16}$ Raman spectroscopic investigations reveal characteristic $D$ and $G$ peaks in all the samples. Additional features observed in the vicinity of $1135-1200 \mathrm{~cm}^{-1}$ are indicative of a structure that mainly contains aromatic rings interspersed with polymeric chains of carbon atoms with single and double bond alternation. Neutron diffraction studies ${ }^{8}$ also confirm the above conclusion. The structural features seen in these films are unusual when compared with other forms of amorphous carbon.

\section{ACKNOWLEDGMENTS}

The authors thank Professor M. S. Hegde and Dr. M. Sahana for their help in the XRD experiments and Richard S. Myer of Astex Co. for his help in obtaining the Raman spectra. One of the authors (V. M.) gratefully acknowledges the insightful comments of Dr. R. A. Narayanan. This work was supported by Department of Science and Technology, and Council for Scientific and Industrial Research, India.

\footnotetext{
${ }^{1}$ Supercarbon: Synthesis, Properties and Applications edited by S. Yoshimura and R. P. H. Chang (Springer-Verlag, Berlin, 1998).

${ }^{2}$ M. L. Kaplan, P. H. Schmidt, C.-H. Chen, and W. M. Walsh, Appl. Phys. Lett. 36, 867 (1980).

${ }^{3}$ M. L. Kaplan, S. R. Forrest, P. H. Schmidt, and T. Venkatesan, J. Appl. Phys. 55, 732 (1984).
} 
${ }^{4}$ M. N. Vijayashree and S. V. Subramanyam, Solid State Commun. 79, 629 (1991).

${ }^{5}$ A. Sayeed, S. Bhattacharyya, and S. V. Subramanyam, Mater. Sci. Eng., C 3, 231 (1995).

${ }^{6}$ V. Meenakshi and S. V. Subramanyam, Int. J. Mod. Phys. B 14, 224 (2000).

${ }^{7}$ V. Meenakshi and S. V. Subramanyam (unpublished).

${ }^{8}$ P. S. R. Krishna, P. Balaya, B. A. Dasannacharya, A. Sayeed, V. Meenakshi, and S. V. Subramanyam, Physica B 241-243, 921 (1998).

${ }^{9}$ Y. Ohki, in Supercarbon: Synthesis, Properties and Applications, edited by S. Yoshimura and R. P. H. Chang (Springer-Verlag, Berlin, 1998), p. 159.

${ }^{10}$ S. V. Subramanyam, A. Sayeed, V. Meenakshi, A. Cholli, and S. Tripathi, J. Appl. Phys. 81, 2907 (1997).

${ }^{11}$ B. E. Warren, X-Ray Diffraction (Dover, New York, 1990).

${ }^{12}$ O. E. Anderson, B. L. V. Prasad, H. Sato, T. Enoki, Y. Hishiyama, Y. Kaburagi, M. Yoshikawa, and S. Bandow, Phys. Rev. B 58, 16387 (1998).

${ }^{13}$ A. C. Ferrari and J. Robertson, Phys. Rev. B 61, 14095 (2000).

${ }^{14}$ B. Bouchet, J. Dixmier, T. Heitz, J. E. Bouree, and C. Godet, J. Non-Cryst. Solids 266-269, 755 (2000).

${ }^{15}$ Y. Maniwa, Y. Kumazawa, Y. Saito, H. Tou, H. Kataura, H. Ishii, S. Suzuki, Y. Achiba, A. Fujiwara, and H. Suematsu, Jpn. J. Appl. Phys., Part 2 38, L668 (1999).

${ }^{16}$ J. H. Kim, J. H. Kim, H. K. Sung, H. J. Kim, C. O. Yoon, and H. Lee, Synth. Met. 84, 71 (1997).

${ }^{17}$ E. Hoinkis, in Chemistry and Physics of Carbon, Vol. 25, edited by P. A. Thrower (Marcel Dekker, New York, 1997); G. M. Jenkins and K. Kawamura, Polymeric Carbons-Carbon Fibre, Glass and Char (Cambridge University Press, Cambridge, England, 1976); F. Li and J. S. Lannin, Phys. Rev. Lett. 65, 1905 (1990).

${ }^{18}$ S. Kugler, K. Shimakawa, T. Watanabe, K. Hayashi, I. László, and R. Bellissent, J. Non-Cryst. Solids 164, 1163 (1993).

${ }^{19}$ S. R. Elliot, Physics of Amorphous Materials, 2nd ed. (Longman Scientific \& Technical, Essex, 1990).

${ }^{20}$ R. O. Dillon, J. A. Woollam, and V. Katkanat, Phys. Rev. B 29, 3482 (1984).
${ }^{21}$ M. Yoshikawa, G. Katagiri, H. Ishida, A. Ishitani, and T. Akamatsu, J. Appl. Phys. 64, 6464 (1988); M. A. Tamor, J. A. Haire, C. H. Wu, and K. C. Hass, Appl. Phys. Lett. 54, 123 (1989).

${ }^{22}$ J. Robertson, Prog. Solid State Chem. 21, 199 (1991); J. Robertson, Adv. Phys. 35, 317 (1986), and references therein.

${ }^{23}$ N. Wada, P. J. Gaczi, and S. A. Solin, J. Non-Cryst. Solids 35-36, 543 (1980)

${ }^{24}$ S. Prawer, F. Ninio, and I. Blanchonette, J. Appl. Phys. 68, 2361 (1990).

${ }^{25}$ S. M. Mominuzzaman, K. M. Krishna, T. Soga, T. Jimbo, and M. Umeno, Carbon 38, 127 (2000).

${ }^{26}$ G. Messina, A. Paoletti, S. Santangelo, A. Tagliaferro, and A. Tucciarone, J. Appl. Phys. 89, 1053 (2001).

${ }^{27}$ I. Harada, Y. Furukawa, and M. Tasumi, J. Chem. Phys. 73, 4746 (1980); F. B. Schugerl and H. Kuzmany, ibid. 74, 953 (1981); L. Rimai, M. E. Heyde, and D. Gill, J. Am. Chem. Soc. 95, 4493 (1973); H. Takeuchi, Y. Furukawa, and I. Harada, J. Chem. Phys. 80, 4746 (1985)

${ }^{28}$ H. Watanabe, K. Takahashi, and M. Iwaki, Nucl. Instrum. Methods Phys. Res. B 80/81, 1489 (1993); H. Watanabe, K. Takahashi, S. Sato, and M. Iwaki, in Proceedings of the Fourteenth Symposium on Ion Sources and Ion-Assisted Technology, edited by T. Takagi (Tokyo, 1991), p. 385.

${ }^{29}$ A. C. Ferrari and J. Robertson, Phys. Rev. B 63, 121405(R) (2001).

${ }^{30}$ F. Li and J. S. Lannin, Appl. Phys. Lett. 61, 2116 (1992).

${ }^{31}$ V. Meenakshi, S. V. Subramanyam, A. Cholli, and S. Tripathy, in Second International Specialists Meeting on Amorphous Carbon, edited by X. Shi, S. R. P. Silva, W. I. Milne, and G. A. J. Amaratunga (1999), p. 31.

${ }^{32}$ J. M. Guet and D. Tchoubar, Fuel 65, 107 (1986).

${ }^{33}$ M. Murakami, S. Iijima, and S. Yoshimura, J. Appl. Phys. 60, 3856 (1986).

${ }^{34}$ M. F. Thorpe, J. Non-Cryst. Solids 57, 355 (1983).

${ }^{35}$ P. Boolchand, M. Zhang, and B. Goodman, Phys. Rev. B 53, 11488 (1996).

${ }^{36}$ J. A. Theil, J. Vac. Sci. Technol. B 17, 2397 (1999).

${ }^{37}$ J. C. Phillips, Philos. Mag. B 79, 527 (1999). 